\title{
Unraveling molecular mechanism underlying biomaterial and stem cells interaction during cell fate commitment using high throughput data analysis
}

\author{
Erfan Sharifi ${ }^{1}$, Niusha Khazaei ${ }^{2}$, Nicholas Kieran ${ }^{2}$, Sahel Jahangiri Esfahani ${ }^{3}$, \\ Abdulshakour Mohammadnia ${ }^{2}$, and Moein Yaqubi ${ }^{2}$ \\ ${ }^{1}$ Islamic Azad University Science and Research Branch \\ ${ }^{2}$ McGill University \\ ${ }^{3}$ Shahrekord University
}

May 7, 2020

\begin{abstract}
Stem cell (SC) differentiation towards somatic cells has proven to be an effective technique in the understanding and progression of regenerative medicine. Despite improvements, concerns regarding the efficiency of differentiation and the differences between $\mathrm{SC}$ products and their in vivo counterparts must be addressed. Biomaterials that mimic endogenous growth conditions represent one recent method used to improve the quality and efficiency of SC differentiation. Here, we aim to use bioinformatics approaches to accomplish two aims: 1) determine the effect of different biomaterials on SC growth and differentiation, and 2) understand the effect of cell of origin on the differentiation potential of multipotent SCs. First, we demonstrate that the dimensionality $(2 \mathrm{D}$ versus $3 \mathrm{D})$ and the degradability of biomaterials affects the way that the cells are able to grow and differentiate at the transcriptional level. Additionally, the particular cell of origin is an important factor in determining the response of SCs to same biomaterial transcriptionally. Our data demonstrates the ability of bioinformatics to understand novel molecular mechanisms and context by which SCs are most efficiently able to differentiate. These results and strategies may suggest proper combinations of biomaterials and SCs to achieve high differentiation efficiency and functionality of desired cell types.
\end{abstract}

\section{Introduction}

Stem cells are defined as having the capacity for self-renewal and the potential to generate multiple types of differentiated cells. These two qualities provide a fantastic foundation for stem cells to benefit the field of regenerative medicine. In the early days of stem cell biology, researchers were challenged by ethical issues and also by limited access and the low number of embryonic stem cells (ESCs) available (Volarevic et al., 2018). This difficulty was overcome with the generation of induced pluripotent stem cells (iPSCs) (Takahashi et al., 2007; Takahashi \& Yamanaka, 2006). iPSCs possess the ability to be derived from easily accessible cells, allowing their collection in big numbers while avoiding ethical concerns associated with ESCs (Takahashi et al., 2007; Takahashi \& Yamanaka, 2006). Other limitations of stem cell efficacy have arisen that must be addressed to tap the full potential of these cells. iPSC differentiation to target cell types can be a laborious process that takes weeks or months depending on the cell type. Once the differentiation process is complete, the produced cells often have a low efficiency of complete differentiation and a limited resemblance to their natural counterparts (Cahan et al., 2014; Tabar \& Studer, 2014). Although there are many contributors to the differentiation result of iPSCs, the microenvironment of the cells is an important player in the successful outcome of differentiation. Media-derived factors, primarily cytokines, as well as the specific material on which the cells grow, impact the success of maturation. Biomaterials act as a beneficial growth platform for cells and may help in the differentiation of iPSCs (Dawson, Mapili, Erickson, Taqvi, \& Roy, 2008). 
Biomaterials are compounds that interact with biological systems, thus impacting the growth and health of cells around them. A combination of ideal media and proper growth platform is an exciting prospect to increase the similarity between iPSC derived cells and theirin vivo counterparts.

Biomaterials have been used for targeted differentiation to generate a variety of cell types. For example, human adipose-derived adult stem cells grown on biomaterials like alginate, agarose hydrogels, and porous gelatin scaffolds can effectively be differentiated towards chondrocytes (Awad, Wickham, Leddy, Gimble, \& Guilak, 2004). Moreover, it was indicated in 2011 that thiolated-hyaluronic acid (HA) hydrogels crosslinked with polyethylene glycol diacrylate appeared to mimicin vivo tissue stiffness and resulted in the generation of more mature cardiomyocytes than previous matrices used (Young \& Engler, 2011). This finding highlights the role of biomaterials as factors to improve the quality of differentiation in generating more mature cells (Young \& Engler, 2011). Another example was highlighted in 2009 in which Smith and colleagues indicated that the use of nanofibrous gelatin/apatite can simulate the normal bone extracellular matrix (ECM) and subsequently increase the differentiation efficiency of stem cells towards an osteogenic fate (Smith, Liu, Hu, Wang, \& Ma, 2009). Since these initial examples confirmed biomaterials to be important contributors to proper stem cell differentiation, a wide range of biomaterials have been used for a variety of cell types. These include polystyrene, graphene, nano-structured hydroxyapatite (HAp), fibrin, poly-lactic-co-glycolicacid (PLGA), and self-gelling alginate, used to generate neuronal stem cells, astrocytes, adipocytes, osteoblasts, cardiomyocytes, and chondrocytes (Bagheri-Hosseinabadi, Mesbah-Namin, Salehinejad, \& Seyedi, 2018; Jakobsen, Ostrup, Zhang, Mikkelsen, \& Brinchmann, 2014; Jeon et al., 2016; Leong et al., 2016; N. Li et al., 2013; Tay et al., 2010). Despite extensive studies using biomaterials to direct stem cells towards a specific fate, the most effective biomaterial for each circumstance remains unknown. Additionally, many of the mechanisms of effectiveness remain to be elucidated, making it difficult to logically progress from one biomaterial to another.

Although the specific effect of different biomaterials on cellular development may be unclear, it is evident that particular biomaterials have a significant impact on the proper differentiation of cells. The choice of biomaterial induces complex biological changes in cells, namely at the transcriptomic level, that will ultimately affect cell fate. The advent of high throughput transcriptomic technologies like microarray, bulk RNA-sequencing (RNA-seq), and single cell RNA-seq (scRNA-seq) allows us to understand these complex cellular changes. Several groups have applied high throughput transcriptomic data analysis to assess the effect of biomaterials on stem cells (Jaager, Islam, Zajac, Linnarsson, \& Neuman, 2012; Kim et al., 2016; Q. Li et al., 2018; Roson-Burgo, Sanchez-Guijo, Del Canizo, \& De Las Rivas, 2014; Yeung et al., 2015). Knight and colleagues found that culturing a human astrocytic cell line on the hydrogel PuraMatrix can induce neuronal transformation of cultured cells, as evidenced by a functional shift of these cells towards neurons (Knight \& Serrano, 2017). Also, in 2011, the effect of two different long-term degradable materials, PLLA-coTMC (Resomer@ LT706) and poly- $\varepsilon$-caprolactone, on the differentiation of mesenchymal stem cells (MSCs) toward osteogenic fate was assessed (Neuss et al., 2011). The results showed that Resomer@ LT706 is the more potent biomaterial in inducing osteogenic fate through increasing the expression of genes involved in bone development, resulting in MSC derived cells that share a greater similarity with endogenous cells (Neuss et al., 2011). Despite the availability of sophisticated transcriptomic technologies, bioinformatics, and systems biology tools, the field of stem cells and biomaterials is not well connected. As detailed above, many groups have used high throughput techniques to analyze stem cell and biomaterial interaction. That being said, these analyses generally skim the surface of stem cell differentiation effectiveness. The use of bioinformatics and systems biology tools to look at interactions of gene regulatory networks, key biological processes, and transcription factors implicated in differentiation processes will provide a deeper understanding of both stem cells and of biomaterials.

In this study, we investigated the interaction between different biomaterials and stem cells, as well as the differentiation potential of stem cells derived from a particular cell of origin. Both questions were addressed using high throughput transcriptomic data analysis and application of systems biology tools. Overall, we showed that the type of the biomaterial used, and the origin of stem cells are both key contributors to the successful differentiation process of stem cells by affecting the transcriptome. We hope that this study 
emphasizes the importance of biomaterial and cell-of-origin selection to optimize stem cell differentiation. These types of analyses highlight the importance of integrating the fields of biomaterials and stem cells through the use of bioinformatics analysis.

\section{Material and Methods:}

\section{Transcriptome data analysis}

All gene expression data set with gene accession numbers GSE46315, GSE85391, and GSE13715 were obtained from gene expression omnibus (GEO) database (de Peppo et al., 2013; Gjorevski et al., 2016; Lai, Asthana, Cheng, \& Kisaalita, 2011). For microarray data sets, in addition to the normalized data, all annotation files in the GPL formats were downloaded from the same database. Annotated gene expression data sets were uploaded into NetworkAnalyst web-tool (Xia, Gill, \& Hancock, 2015; Zhou et al., 2019). For unnormalized data, $\log 2$ transformation was used to perform normalization and Limma algorithm was applied as a statistical algorithm to calculate differentially expressed genes (DEGs) (Xia et al., 2015; Zhou et al., 2019). As a general rule for all data sets, we used adjusted $p$-value $<0.05$ and $\log 2$ fold change $>1$ to identify DEGs. The list of highly affected genes with adjusted $p$-values $<0.05$ and $\log 2$ fold change $>1$ were used to draw Venn diagrams.

In addition to microarray data sets, we used a Mouse RNA-sequencing data set with accession number GSE85391. Read count matrix of this study was downloaded from GEO database (Gjorevski et al., 2016)and was analyzed with edgeR package in $\mathrm{R}$ to find differentially expressed genes (Robinson, McCarthy, \& Smyth, 2010). DEGs were filtered using edgeR adjusted $p$-values $<0.05$ and $\log 2$ fold change $>1$.

\section{Biological process analysis:}

To identify the most affected biological process three types of ontology analysis was done in this study. First, Gene Set Enrichment Analysis (GSEA) tool was used to highlight overrepresented genes and enriched pathways (Mootha et al., 2003; Subramanian et al., 2005). GSEAPreranked analysis tool was recruited for which we used the list of differentially expressed genes which had been ordered based on their fold changes. We used biological process gene ontology and decreased minimum set size from 15 to 1 (Mootha et al., 2003; Subramanian et al., 2005). Second, we used Enrichr webtool to dissect data in more detail. In this case, the most affected biological processes were filtered and sorted based on their adjusted $p$-value and number of differentially expressed genes (Chen et al., 2013; Kuleshov et al., 2016). Third, we did biological processes ontology on the constructed gene regulatory network in Cytoscape software using ClueGO and CluePedia plugins. In the ClueGO and CluePedia plugins advanced statistical option of the tools, two-sided hypergeometric test was selected to calculate the importance of each term and Bonferroni step-down was used for p-valuecorrection (Bindea, Galon, \& Mlecnik, 2013; Bindea et al., 2009). The terms with p-value $<0.05$ were considered as meaningful terms.

\section{Construction and analyzing integrated gene regulatory network:}

The data of transcription factors (TF)-binding site were obtained from the ChIP Enrichment analysis (ChEA) database (Chen et al., 2013; Kuleshov et al., 2016).ChEA contains TF-DNA interactions data obtained from ChIP-chip, ChIP-seq, ChIP-PET, and DamID (Chen et al., 2013; Kuleshov et al., 2016). We perused the DEGs list in this database and retrieved TFs that controlled the expression of DEGs and kept TFs that contained $p$-value $<0.05$. In the case of TFs, instead of our criteria to find DEGs (log2 fold change $>1)$ as a common fold change, we set another level of filtering to identify differentially expressed TFs (DE-TFs) by setting expression fold change to 1.5 and selecting those as DE-TFs. Using TF-binding site data of the DE-TFs, DE-TF expression, and DEGs, the GRNs were constructed in the Cytoscape software. The most central TFs which regulate majority of the downregulated genes were identified using CentisCape plugin of the Cytoscape (Scardoni, Petterlini, \& Laudanna, 2009). This plugin considers the direct interaction of the TFs with their targets as parameter called degree. Those TFs with highest number of target genes will be considered as the most central TFs.

\section{Protein complex analysis}


To predict the most important protein complexes, protein-protein interactions for differentially expressed TFs (DE-TFs) were retrieved from STRING database (Szklarczyk et al., 2019). These interactions were filtered by transcriptomic data to keep only meaningful interactions. Finally these interactions along with expression data were loaded into the cystoscope software (Saito et al., 2012) and a protein-protein interaction network was constructed. This network was analyzed by MCODE software (Bader \& Hogue, 2003) in the Cytoscape software environment. Complexes with scores more than two were considered as significant protein complexes.

\section{PCA and heatmap generation:}

Principal component analysis was achieved using the built-in $\mathrm{R}$ function prcomp and ploted using ggplot2 package in R. Microarray normalized intensities were used to generate heatmap and visualize using "Superheat" package in R (Barter \& Yu, 2018).

\section{Results:}

In order to study the role of biomaterials in stem cell differentiation, we analyzed public transcriptomic data sets generated from either microarray or RNA-seq. First, we highlight the importance of biomaterial dimensionality, as we evaluated the effect of $2 \mathrm{D}$ versus $3 \mathrm{D}$ scaffolds on neural progenitor cell differentiation. Furthermore, we evaluate the impact of biomaterial degradability on the efficiency of stem cell differentiation. Finally, we study how mesenchymal stem cells originated from different pluripotent stem cells result in osteoblasts with different transcriptomic profiles.

The effect of different scaffolds on the differentiation of a single cell type: $2 \mathrm{D}$ and $3 \mathrm{D}$ scaffolds on neural progenitor cells differentiation.

A longstanding difference between in vitro and in vivocell growth conditions is the dimensionality difference between the two.In vitro experiments have traditionally used two-dimensional cell monolayers (discounting recent organoid developments), while cells in live organisms develop in a three-dimensional setting. The dimensionality of a cell's microenvironment can have profound effects on the function and differentiation of said cell (Willerth \& Sakiyama-Elbert, 2019). Transcriptomic responses can be documented using highthroughput techniques like microarray and RNA sequencing.

In 2011, Lai et al used 2D and 3D porous polystyrene scaffolds to assess and compare the effect of dimensionality on neural progenitor cell (NPC) differentiation (Lai et al., 2011). NPCs were maintained in neural basal media supplemented with penicillin, L-glutamine, recombinant human leukemia inhibitory factor, basic fibroblast growth factor (bFGF) and B-27 (Lai et al., 2011). Later on, bFGF was removed from above mentioned factors to start differentiation process ${ }^{25}$. NPCs cultured in the presence of the $2 \mathrm{D}$ substrate showed significant transcriptomic remodeling with 890 DEGs, with 439 of these genes up-regulated (FC $>2$ and $p$-value $<0.05$ ). The differentiated NPCs were clearly segregated from undifferentiated cells (Fig. 1a). The most statistically significant upregulated genes were those related to the central nervous system (CNS), including nervous system development, axon guidance, and axonogenesis. (Fig. 1b, Table S1). The same type of analysis with the same criteria $(\mathrm{FC}>2$ and $p$-value $<0.05)$ for cells grown in a $3 \mathrm{D}$ scaffold led us to find 1082 DEGs, most of which were upregulated. The differentiated cells in presence of the 3D substrate also were separated from undifferentiated cells (Fig1a). The analysis of biological processes similarly showed neuronal differentiation related terms as the most significantly upregulated terms (Fig. 1c, Table S2). We then compared the list of DEGs between each scaffold dimension to elucidate the differences between cultures. The results of this comparison revealed a high level of transcriptome similarity between culturing neural progenitor cells in 2D and 3D scaffolds (Fig. 1d). 52\% of DEGs in the 2D scaffold dataset were differentially expressed with the same expression pattern in the presence of the 3D scaffold. As expected, 480 common DEGs found in both 2D and 3D scaffold data sets were involved in nervous system related biological processes and functions, like neuron differentiation (FDR $q$-val $=0.0046)$ (Fig. 1e and 1f, Table S3). Despite similarities, the expression of many genes was different between 2D and 3D scaffolds, with many more upregulated genes in the 3D culturing condition. Because tissue cells grow in a 3D structure, we hypothesized that neuronal cells differentiated on the $3 \mathrm{D}$ scaffold would be more similar to their in vivo 
counterparts. Therefore, we expected that these up-regulated genes in the 3D culturing condition would be associated with nervous system development. We performed ontology analysis for the most upregulated genes that were exclusive to the $2 \mathrm{D}$ biomaterial and we found that the most affected terms were related to nervous system development and functions, including synapse assembly, chemical synaptic transmission, and anterograde trans-synaptic signaling (Fig. 1g). We performed the same analysis for the most upregulated genes exclusive to the 3D biomaterial, and unexpectedly found a high number of terms associated with inflammatory response (Fig. 1h). The $2 \mathrm{D}$ most upregulated terms lacked any that were related to immune function. Overall, the transcriptomic profile of neural progenitor cells cultured in 2D and 3D scaffolds revealed that both induce common gene expression related to nervous system development. In addition to these genes, however, the 3D scaffold induces more biological processes that are not related to nervous system development, including inflammatory responses. Therefore, in contrast to our hypothesis, it seems culturing of NPCs on 2D porous polystyrene produce neurons with more mature cell characteristics according to their transcriptome profile, however, further experimental studies are needed to confirm our result in more detail.

Dimensionality is an important contributor to stem cell differentiation and relatedness to in vivo counterparts. However, there are other modalities of biomaterials that can influence the success of differentiation. Here, we investigate the role of degradable vs non-degradable biomaterials as one influencer. Gjorevski et al. used either degradable or non-degradable enzymatically crosslinked polyethylene glycol (PEG) during epithelial organoid formation from intestinal stem cells (ISCs) (Gjorevski et al., 2016). Remodeling is important for repair and morphogenesis, so they hypothesized that the degradable matrix would improve organoid formation. Interestingly, their results indicated that the use of degradable PEG resulted in an abnormal shape of colonies and fewer organoids formed. The transcriptomic profile of ISCs demonstrated that genes related to stress and inflammation were significantly enriched in ISCs cultured in the presence of degradable PEG compared to non-degradable PEG. We re-analyzed their RNA-seq data to further investigate the possible mechanism of the poor organoid formation from ISCs cultured in the degradable PEG.

Comparing ISC differentiation in the presence of degradable and non-degradable PEGs showed 170 DEGs $(\mathrm{FC}>2$ and $p$-value $<0.05)$. Among these, 115 genes were upregulated in degradable PEGs, and the majority were associated with an inflammatory response and a positive regulation of leukocyte chemotaxis in comparison with non-degradable PEGs (Fig. 2a). To find the main regulators of the inflammatory response related genes in ISCs differentiation, we generated a gene regulatory network containing 33 genes (nodes) and 53 interactions between them (edges) (Fig. 2b). Analysis of this constructed gene regulatory network revealed ATF3 and KLF6 as the main regulators of the inflammatory response (Fig. 2c). In addition, we constructed another network using a list of differentially expressed TFs, called protein-protein interaction network (Fig. 2d). In the constructed protein-protein interaction network, ATF3 and KLF6 are found to interact with NFKBIA that subsequently interacts with TNF. Interestingly, it was previously shown that KLF6 expression dramatically increased in both intestinal tissue and myeloid cells in inflammatory bowel disease (Goodman et al., 2016). So, it seems that recruitment of transcription factors ATF3 and KLF6 could be a possible mechanism for activation inflammatory cascaded in abnormal organoid formation.

The differentiation potential of stem cells from different origins: Osteogenic differentiation from iPSCs, ESCs, and BMSC-derived mesenchymal stem cells.

Cellular context affects the way that cells respond to different biomaterials. Although stem cells from different sources retain the capacity to divide and differentiate endlessly, each source imprints subtle changes to cells that affect their differentiation process. To address how stem cells from different sources respond to the same biomaterial, we used data generated from mesenchymal stem cells (MSCs) under differentiation on osteoinductive scaffolds in perfusion bioreactors (de Peppo et al., 2013). These MSCs arose from three different origins: they were differentiated from human induced pluripotent stem cells (hiPSCs) or human embryonic stem cells (hESCs), or they were directly acquired from human bone marrow (BM) (de Peppo et al., 2013). All these different cells were induced to MSCs using serum-supplemented medium for 7 days and then more 10-11 passages (de Peppo et al., 2013). The main focus of that study was proving the feasibility of using hiPSCs for generation of osteoblasts. 
We used this dataset to more deeply analyze the transcriptomic differences between osteoblast differentiation of different cells of origin. For hiPSC, hESC and bone marrow cells, we compared gene expression profiles of MSCs before and after differentiation towards osteoblasts. In summary, using the same criteria (FC $>2$ and adjusted $p$-value $<0.05)$ we found 416,869, and 341 differentially expressed genes respectively for hiPSCs, hESCs, and BM-derived MSCs differentiated into osteogenic fate (Fig. S1). Analysis of the common genes in transcriptomic level from all differentiation experiments using osteoinductive scaffolds in perfusion bioreactors showed an up regulation of an osteoblast specific profile as the most significant cell signature (Fig. 3a and 3b, Table S4). Many of these genes are involved in extracellular matrix organization, including COL12A1, COL8A2, and COMP. It has been shown that COL12A1 is involved in regulation of osteoblast differentiation and bone matrix formation (Izu et al., 2011). This suggests that MSCs generated from different stem cells can successfully be committed into osteogenic fate. Despite the similarities between all induced osteoblasts, we found that differentially expressed genes during osteogenic differentiation of ESC derived and iPCS derived MSCs clustered together but were markedly different from osteogenic differentiation with BM origin (Fig. S2).

To get a better understanding of osteogenic differentiation from different cells of origin, we restricted our list to genes that were exclusively upregulated in cells differentiated from hESC, hiPSC, or BM. There were 549,197 , and 134 genes significantly upregulated $(\mathrm{FC}>2$ and $p$-values $<0.05)$ from hESC-MSC, hiPSCMSC, and BM-MSC derived osteoblasts, respectively. Gene set enrichment analysis showed genes involved in extracellular matrix organization enriched significantly in iPSC-MSC and ESC-MSC derived osteoblasts, but not in BM-MSC derived osteoblasts (Fig. 3c and 3d). In comparing genes involved in extracellular matrix organization between iPSCs and ESCs, we found higher number of genes with more significant $p$ value related to this biological term in iPSCs than ESCs. Extracellular matrix as a local microenvironment of osteoblast has significant role in cell motility, communication and shape by providing an anchorage site for cells and storing and presenting cytokines for cell growth (Rozario \& DeSimone, 2010).

To provide a better understanding of the different differentiation potentials of MSCs from each origin, we made a gene regulatory network for genes exclusively involved in the differentiation of ESC-MSCs into osteoblasts. We then mapped gene expression profiles of osteoblasts generated from all three cells of origin onto the ESC-MSC constructed gene regulatory network (Fig. 3e, 3f, and 3g). Our results clearly indicated these three cells of origin showed significantly different responses in the gene expression level upon osteogenic differentiation. For example, based on the gene regulatory network analysis, we found the transcription factor MYC to be exclusively down-regulated in the ESC-MSC osteogenic differentiation according to adjusted $p$ value of the genes. MYC is a master regulator of cell proliferation and stemness, so this suggests that the ESC-MSC derived osetoblasts were more differentiated than the iPSC and BM derived MSCs. Additionally, $76 \%$ of down-regulated genes in the ESC-MSC network are controlled by MYC, further suggesting that the entire MYC pathway is dampened in the ESC-MSC derived osteoblasts. Network based ontology revealed biological processes related to cell cycle, mitochondrial and metabolism as most significant processes (Fig. $3 h)$.

Our results clearly showed that MSCs originated from different cells of origin were transcriptionally differently respond to the osteogenic differentiation. Despite the fact that all three stem cell populations could differentiate into osteogenic lineage, it is evident that each cell of origin resulted in a moderately distinct transcriptional profile of the differentiated cell. Overall, through bioinformatic analysis of biomaterial-specific responses, as well as analysis of cell-of-origin based response, we highlight that both of these modalities are instrumental in the optimization of targeted stem cell differentiation.

\section{Discussion:}

In this study we highlighted the potential of bioinformatic, and systems biology tools and approaches to provide a better insight into the interaction of biomaterials with stem cells. Bioinformatic analyses can unravel subtle molecular interactions between biomaterials and stem cells. Using such information will allow for the optimization of stem cell differentiation in regard to time, expense, and quantity of properly differentiated cells. Here, we briefly discuss the impact of biomaterials and of cells of origin on stem cell 
differentiation.

Recent studies have shown that the microenvironment in which stem cells grow has a dramatic effect on the efficiency of generated cells (Tibbitt \& Anseth, 2012). For example, it has been shown that hyaluronic acid-based 3D hydrogels can successfully be used to culture hippocampal neural progenitor cells (Tarus et al., 2016). However, use of a 3D system has limitations, as this dimensionality can negatively impact the size and shape of 3D spheres. For instance, Hydrogel films of 3-hydroxybutyrate and 3-hydroxyhexanoate copolymers has been used in 2D form to drive NSCs toward neurons (Xu et al., 2010). This is one example in which the ideal culturing techniques have yet to be completely elucidated, stressing the importance to better understand 2D versus 3D culturing systems. In our study, we delved deeper into the importance of biomaterial dimensionality for NPC differentiation and found that NPCs cultured on 2D porous polystyrene scaffolds appear to produce more transcriptionally mature neuronal cells than those cultured on 3D biomaterials based on their transcriptome profile. Our data also indicates the importance of specific biomaterial use, as NPCs were differentiated more efficiently on a 3D hyaluronic acid compared to 2D, but both of these results were overshadowed by the efficiency of NPC differentiation on 2D porous polystyrene scaffolds.

In addition to the effect of biomaterials on stem cell differentiation, the cell of origin has been shown to be an important factor in determining the quality of the differentiated cell. For example, Wu et al. compared biological characteristics of different adult multipotent stem cells, including stem cells isolated from human bone marrow, placental decidua basalis and urine, based on their ability in colony formation, morphology, proliferation and differentiation into different cell types (Wu et al., 2018). They showed that BM-MSCs have a higher potential to differentiate into chondrogenic and osteogenic cell lineages than other multipotent stem cells (Wu et al., 2018). In another study, Rim et al. took a similar approach, but they aimed to determine the effect of the cell of origin on iPSC differentiation. iPSCs were generated from four different cell of origins, including dermal fibroblasts (DF), peripheral blood mononuclear cells (PBMC), cord blood mononuclear cells (CBMC), and osteoarthritis fibroblast-like synoviocytes (OAFLS) (Rim et al., 2018). Differentiation of these cells toward chondrogenic fate showed iPSCs derived from CBMC had the highest differentiation potential (Rim et al., 2018). These groups found that cell of origin played an important role in the functionality of differentiated cells. However, we believed that there are more subtle differences between cells differentiated from separate cells of origin that may be difficult to interpret with some functional assays. Therefore, we investigated differences at the transcriptomic level that would give a more precise similarity/difference readout between the different cells of origin as they were differentiated.

Therefore, we attempted to determine whether MSCs generated from different cell sources, including hiPSCs, hESCs, and BM, have different capacities during osteogenesis. Our results show that osteoblasts generated from hiPSC-MSCs and hESC-MSCs clustered together, while BM-MSC derived osteoblasts displayed a greater variability in their transcriptomic profile. Furthermore, our results clearly showed more genes involved in cell cycle and extracellular matrix organization in differentiated osteoblasts from MSCs originated from ESCs and iPSCs than BM-MSCs. The transcriptomic profile of each separately derived osteoblast lineage was mapped on the regulatory network specific for ESC-MSCs, and large differences between each lineage were evidently observed. In this regulatory network, MYC was significantly decreased exclusively in the ESC-MSC generated osteoblast and was the main regulator of the entire network in this lineage. Ontology analysis of MYC target genes also showed that cell cycle and mitochondrial terms were the most significant affected biological terms. This finding is of interest because Hanna et al and Persson et al showed that the proliferative capacity of osteogenic differentiating cells reduces in later stages of this process (Hanna, Mir, \& Andre, 2018; Persson et al., 2018). Therefore, according to the expression pattern of MYC in osteoblasts generated across the three cells of origin, the ESC-MSC derived osteoblasts may be the most biologically relevant differentiated cell. Although more analysis is required to get the full picture, our analysis shows the importance of looking at the transcriptomic level of different cells of origin to understand which lineage may be the best for a particular differentiation.

\section{Conclusion}

In summary, we highlighted the importance of applying bioinformatics analysis and systems biology tools to 
the fields of stem cells and biomaterials. The application of biomaterials is rapidly expanding as a mechanism to impact cellular differentiation, yet there remains a significant gap in our understanding of the molecular changes that biomaterials induce. Further in silico analyses are required to more aptly define the role of biomaterials in stem cell growth. Our results begin to make this characterization, as we demonstrated the change in differentiation of cells grown on different biomaterials at the transcriptomic level. Finally, we highlighted the importance of application of bioinformatics approach in understanding stem cells and biomaterials interactions in order to propose a strategy to achieve higher differentiation efficiency and quality.

Conflict of interest: The authors declare that they have no conflicts of interest with the contents of this article.

Acknowledgments: This research did not receive any external funding and was conducted using the authors' personal money.

Author Contributions: Data collection, multi-omics data analysis, gene expression experiments: ES, NK, SJE, MY, and AM. Designed the experiments: ES, NK, MY, and AM. Wrote the paper: ES, NK, NWK, $\mathrm{MY}$, and AM.

\section{References}

Awad, H. A., Wickham, M. Q., Leddy, H. A., Gimble, J. M., \& Guilak, F. (2004). Chondrogenic differentiation of adipose-derived adult stem cells in agarose, alginate, and gelatin scaffolds. Biomaterials, 25 (16), 3211-3222. doi:10.1016/j.biomaterials.2003.10.045

Bader, G. D., \& Hogue, C. W. (2003). An automated method for finding molecular complexes in large protein interaction networks. BMC Bioinformatics, 4 , 2. doi:10.1186/1471-2105-4-2

Bagheri-Hosseinabadi, Z., Mesbah-Namin, S. A., Salehinejad, P., \& Seyedi, F. (2018). Fibrin scaffold could promote survival of the human adipose-derived stem cells during differentiation into cardiomyocyte-like cells. Cell Tissue Res, 372 (3), 571-589. doi:10.1007/s00441-018-2799-9

Barter, R. L., \& Yu, B. (2018). Superheat: An R package for creating beautiful and extendable heatmaps for visualizing complex data. J Comput Graph Stat, 27 (4), 910-922. doi:10.1080/10618600.2018.1473780

Bindea, G., Galon, J., \& Mlecnik, B. (2013). CluePedia Cytoscape plugin: pathway insights using integrated experimental and in silico data. Bioinformatics, 29 (5), 661-663. doi:10.1093/bioinformatics/btt019

Bindea, G., Mlecnik, B., Hackl, H., Charoentong, P., Tosolini, M., Kirilovsky, A., . . . Galon, J. (2009). ClueGO: a Cytoscape plug-in to decipher functionally grouped gene ontology and pathway annotation networks. Bioinformatics, 25 (8), 1091-1093. doi:10.1093/bioinformatics/btp101

Cahan, P., Li, H., Morris, S. A., Lummertz da Rocha, E., Daley, G. Q., \& Collins, J. J. (2014). CellNet: network biology applied to stem cell engineering. Cell, 158 (4), 903-915. doi:10.1016/j.cell.2014.07.020

Chen, E. Y., Tan, C. M., Kou, Y., Duan, Q., Wang, Z., Meirelles, G. V., . . . Ma'ayan, A. (2013). Enrichr: interactive and collaborative HTML5 gene list enrichment analysis tool. BMC Bioinformatics, 14 , 128. doi:10.1186/1471-2105-14-128

Dawson, E., Mapili, G., Erickson, K., Taqvi, S., \& Roy, K. (2008). Biomaterials for stem cell differentiation. Adv Drug Deliv Rev, 60 (2), 215-228. doi:10.1016/j.addr.2007.08.037

de Peppo, G. M., Marcos-Campos, I., Kahler, D. J., Alsalman, D., Shang, L., Vunjak-Novakovic, G., \& Marolt, D. (2013). Engineering bone tissue substitutes from human induced pluripotent stem cells. Proc Natl Acad Sci U S A, 110 (21), 8680-8685. doi:10.1073/pnas.1301190110

Gjorevski, N., Sachs, N., Manfrin, A., Giger, S., Bragina, M. E., Ordonez-Moran, P., . . . Lutolf, M. P. (2016). Designer matrices for intestinal stem cell and organoid culture. Nature, 539 (7630), 560-564. doi:10.1038/nature20168 
Goodman, W. A., Omenetti, S., Date, D., Di Martino, L., De Salvo, C., Kim, G. D., . . Mahabeleshwar, G. H. (2016). KLF6 contributes to myeloid cell plasticity in the pathogenesis of intestinal inflammation. Mucosal Immunol, 9 (5), 1250-1262. doi:10.1038/mi.2016.1

Hanna, H., Mir, L. M., \& Andre, F. M. (2018). In vitro osteoblastic differentiation of mesenchymal stem cells generates cell layers with distinct properties. Stem Cell Res Ther, 9 (1), 203. doi:10.1186/s13287-018-0942-x

Izu, Y., Sun, M., Zwolanek, D., Veit, G., Williams, V., Cha, B., . . . Birk, D. E. (2011). Type XII collagen regulates osteoblast polarity and communication during bone formation. J Cell Biol, 193 (6), 1115-1130. doi:10.1083/jcb.201010010

Jaager, K., Islam, S., Zajac, P., Linnarsson, S., \& Neuman, T. (2012). RNA-seq analysis reveals different dynamics of differentiation of human dermis- and adipose-derived stromal stem cells. PLoS One, 7 (6), e38833. doi:10.1371/journal.pone.0038833

Jakobsen, R. B., Ostrup, E., Zhang, X., Mikkelsen, T. S., \& Brinchmann, J. E. (2014). Analysis of the effects of five factors relevant to in vitro chondrogenesis of human mesenchymal stem cells using factorial design and high throughput mRNA-profiling. PLoS One, 9 (5), e96615. doi:10.1371/journal.pone.0096615

Jeon, O. H., Panicker, L. M., Lu, Q., Chae, J. J., Feldman, R. A., \& Elisseeff, J. H. (2016). Human iPSCderived osteoblasts and osteoclasts together promote bone regeneration in 3D biomaterials. Sci Rep, 6 , 26761. doi:10.1038/srep26761

Kim, S. H., Das, A., Chai, J. C., Binas, B., Choi, M. R., Park, K. S., . . . Chai, Y. G. (2016). Transcriptome sequencing wide functional analysis of human mesenchymal stem cells in response to TLR4 ligand.Sci Rep, 6 , 30311. doi:10.1038/srep30311

Knight, V. B., \& Serrano, E. E. (2017). Hydrogel scaffolds promote neural gene expression and structural reorganization in human astrocyte cultures. PeerJ, 5, e2829. doi:10.7717/peerj.2829

Kuleshov, M. V., Jones, M. R., Rouillard, A. D., Fernandez, N. F., Duan, Q., Wang, Z., . . . Ma'ayan, A. (2016). Enrichr: a comprehensive gene set enrichment analysis web server 2016 update. Nucleic Acids Res, 44 (W1), W90-97. doi:10.1093/nar/gkw377

Lai, Y., Asthana, A., Cheng, K., \& Kisaalita, W. S. (2011). Neural cell 3D microtissue formation is marked by cytokines' up-regulation.PLoS One, 6 (10), e26821. doi:10.1371/journal.pone.0026821

Leong, M. F., Lu, H. F., Lim, T. C., Du, C., Ma, N. K. L., \& Wan, A. C. A. (2016). Electrospun polystyrene scaffolds as a synthetic substrate for xeno-free expansion and differentiation of human induced pluripotent stem cells. Acta Biomater, 46 , 266-277. doi:10.1016/j.actbio.2016.09.032

Li, N., Zhang, Q., Gao, S., Song, Q., Huang, R., Wang, L., . . . Cheng, G. (2013). Three-dimensional graphene foam as a biocompatible and conductive scaffold for neural stem cells. Sci Rep, 3 , 1604. doi:10.1038/srep01604

Li, Q., Zhang, B., Kasoju, N., Ma, J., Yang, A., Cui, Z., . . . Ye, H. (2018). Differential and Interactive Effects of Substrate Topography and Chemistry on Human Mesenchymal Stem Cell Gene Expression. Int $J$ Mol Sci, 19 (8). doi:10.3390/ijms19082344

Mootha, V. K., Lindgren, C. M., Eriksson, K. F., Subramanian, A., Sihag, S., Lehar, J., . . . Groop, L. C. (2003). PGC-1alpha-responsive genes involved in oxidative phosphorylation are coordinately downregulated in human diabetes. Nat Genet, 34 (3), 267-273. doi:10.1038/ng1180

Neuss, S., Denecke, B., Gan, L., Lin, Q., Bovi, M., Apel, C., . . . Zenke, M. (2011). Transcriptome analysis of MSC and MSC-derived osteoblasts on Resomer(R) LT706 and PCL: impact of biomaterial substrate on osteogenic differentiation. PLoS One, 6 (9), e23195. doi:10.1371/journal.pone.0023195

Persson, M., Lehenkari, P. P., Berglin, L., Turunen, S., Finnila, M. A. J., Risteli, J., . . . Tuukkanen, J. (2018). Osteogenic Differentiation of Human Mesenchymal Stem cells in a 3D Woven Scaffold. Sci Rep, 8 


\section{(1), 10457. doi:10.1038/s41598-018-28699-x}

Rim, Y. A., Nam, Y., Park, N., Jung, H., Jang, Y., Lee, J., \& Ju, J. H. (2018). Different Chondrogenic Potential among Human Induced Pluripotent Stem Cells from Diverse Origin Primary Cells. Stem Cells Int, 2018 , 9432616. doi:10.1155/2018/9432616

Robinson, M. D., McCarthy, D. J., \& Smyth, G. K. (2010). edgeR: a Bioconductor package for differential expression analysis of digital gene expression data. Bioinformatics, 26 (1), 139-140. doi:10.1093/bioinformatics/btp616

Roson-Burgo, B., Sanchez-Guijo, F., Del Canizo, C., \& De Las Rivas, J. (2014). Transcriptomic portrait of human Mesenchymal Stromal/Stem Cells isolated from bone marrow and placenta. BMC Genomics, 15 , 910. doi:10.1186/1471-2164-15-910

Rozario, T., \& DeSimone, D. W. (2010). The extracellular matrix in development and morphogenesis: a dynamic view. Dev Biol, 341 (1), 126-140. doi:10.1016/j.ydbio.2009.10.026

Saito, R., Smoot, M. E., Ono, K., Ruscheinski, J., Wang, P.-L., Lotia, S., . . . Ideker, T. (2012). A travel guide to Cytoscape plugins. Nature methods, 9 (11), 1069-1076. doi:10.1038/nmeth.2212

Scardoni, G., Petterlini, M., \& Laudanna, C. (2009). Analyzing biological network parameters with CentiScaPe. Bioinformatics, 25 (21), 2857-2859. doi:10.1093/bioinformatics/btp517

Smith, L. A., Liu, X., Hu, J., Wang, P., \& Ma, P. X. (2009). Enhancing osteogenic differentiation of mouse embryonic stem cells by nanofibers. Tissue Eng Part A, 15 (7), 1855-1864. doi:10.1089/ten.tea.2008.0227

Subramanian, A., Tamayo, P., Mootha, V. K., Mukherjee, S., Ebert, B. L., Gillette, M. A., . . Mesirov, J. P. (2005). Gene set enrichment analysis: a knowledge-based approach for interpreting genome-wide expression profiles. Proc Natl Acad Sci US A, 102 (43), 15545-15550. doi:10.1073/pnas.0506580102

Szklarczyk, D., Gable, A. L., Lyon, D., Junge, A., Wyder, S., Huerta-Cepas, J., . . . Mering, C. V. (2019). STRING v11: protein-protein association networks with increased coverage, supporting functional discovery in genome-wide experimental datasets. Nucleic Acids Res, 47 (D1), D607-D613. doi:10.1093/nar/gky1131

Tabar, V., \& Studer, L. (2014). Pluripotent stem cells in regenerative medicine: challenges and recent progress. Nat Rev Genet, 15 (2), 82-92. doi:10.1038/nrg3563

Takahashi, K., Tanabe, K., Ohnuki, M., Narita, M., Ichisaka, T., Tomoda, K., \& Yamanaka, S. (2007). Induction of pluripotent stem cells from adult human fibroblasts by defined factors. Cell, 131 (5), 861-872. doi:10.1016/j.cell.2007.11.019

Takahashi, K., \& Yamanaka, S. (2006). Induction of pluripotent stem cells from mouse embryonic and adult fibroblast cultures by defined factors. Cell, 126 (4), 663-676. doi:10.1016/j.cell.2006.07.024

Tarus, D., Hamard, L., Caraguel, F., Wion, D., Szarpak-Jankowska, A., van der Sanden, B., \& Auzely-Velty, R. (2016). Design of Hyaluronic Acid Hydrogels to Promote Neurite Outgrowth in Three Dimensions. ACS Appl Mater Interfaces, 8 (38), 25051-25059. doi:10.1021/acsami.6b06446

Tay, C. Y., Yu, H., Pal, M., Leong, W. S., Tan, N. S., Ng, K. W., . . . Tan, L. P. (2010). Micropatterned matrix directs differentiation of human mesenchymal stem cells towards myocardial lineage. Exp Cell Res, 316 (7), 1159-1168. doi:10.1016/j.yexcr.2010.02.010

Tibbitt, M. W., \& Anseth, K. S. (2012). Dynamic microenvironments: the fourth dimension. Sci Transl Med, 4 (160), 160ps124. doi:10.1126/scitranslmed.3004804

Volarevic, V., Markovic, B. S., Gazdic, M., Volarevic, A., Jovicic, N., Arsenijevic, N., . . . Stojkovic, M. (2018). Ethical and Safety Issues of Stem Cell-Based Therapy. Int J Med Sci, 15 (1), 36-45. doi:10.7150/ijms.21666 
Willerth, S. M., \& Sakiyama-Elbert, S. E. (2019). Combining Stem Cells and Biomaterial Scaffolds for Constructing Tissues and Cell Delivery.StemJournal, 1 , 1-25. doi:10.3233/STJ-180001

Wu, C., Chen, L., Huang, Y. Z., Huang, Y., Parolini, O., Zhong, Q., . . . Deng, L. (2018). Comparison of the Proliferation and Differentiation Potential of Human Urine-, Placenta Decidua Basalis-, and Bone Marrow-Derived Stem Cells. Stem Cells Int, 2018 , 7131532. doi:10.1155/2018/7131532

Xia, J., Gill, E. E., \& Hancock, R. E. (2015). NetworkAnalyst for statistical, visual and network-based meta-analysis of gene expression data. Nat Protoc, 10 (6), 823-844. doi:10.1038/nprot.2015.052

Xu, X. Y., Li, X. T., Peng, S. W., Xiao, J. F., Liu, C., Fang, G., . . . Chen, G. Q. (2010). The behaviour of neural stem cells on polyhydroxyalkanoate nanofiber scaffolds. Biomaterials, 31 (14), 39673975. doi:10.1016/j.biomaterials.2010.01.132

Yeung, C. Y., Zeef, L. A., Lallyett, C., Lu, Y., Canty-Laird, E. G., \& Kadler, K. E. (2015). Chick tendon fibroblast transcriptome and shape depend on whether the cell has made its own collagen matrix. Sci Rep, 5, 13555. doi:10.1038/srep13555

Young, J. L., \& Engler, A. J. (2011). Hydrogels with time-dependent material properties enhance cardiomyocyte differentiation in vitro.Biomaterials, 32 (4), 1002-1009. doi:10.1016/j.biomaterials.2010.10.020

Zhou, G., Soufan, O., Ewald, J., Hancock, R. E. W., Basu, N., \& Xia, J. (2019). NetworkAnalyst 3.0: a visual analytics platform for comprehensive gene expression profiling and meta-analysis. Nucleic Acids Res, 47 (W1), W234-W241. doi:10.1093/nar/gkz240

\section{Fig. legends:}

Fig. 1. A) Principle Component Analysis (PCA) of the neural progenitor cells and their differentiated neurons on the $2 \mathrm{D}$ and $3 \mathrm{D}$ porous polystyrene. PCA shows the distribution of the samples. $\mathrm{B}$ and $\mathrm{C}$ ) Gene set enrichment analysis of the most up regulated genes in the presence of $2 \mathrm{D}$ and $3 \mathrm{D}$ scaffold respectively. D) Venn diagram shows comparison between the number of differentially expressed genes between $2 \mathrm{D}$ and $3 \mathrm{D}$ scaffolds. E) Gene set enrichment analysis of the common differentially expressed genes between 2D and 3D scaffold. F) Heatmap plot indicating the common differentially expressed genes between 2D and 3D scaffold. Red color shows up regulated genes and green color indicates down regulated ones. G and H) Biological process analysis of the genes that exclusively up regulated in 2D and 3D scaffolds respectively.

Fig. 2. a) biological process analysis of the up regulated genes in the degradable PEGs. The terms are ordered according to their $-\log 10 \mathrm{p}$-value. The number of genes in each term is represented in front of each term. b) Gene regulatory network for the inflammatory response related genes in the intestinal stem cells. Each gene is represented by a node and interaction between them is represented by edge. The red color shows up regulation of the genes and the green one shows down regulation of them. The intensity of the color shows the amount of the expression changes. c) Centrality analysis of the gene regulatory network. Transcription factors are represented in the $\mathrm{x}$ bar and the numbers of interactions are indicated in the $\mathrm{y}$ bar. D) Protein complexes which is obtained from protein-protein interaction network of the differentially expressed TFs.

Fig. 3. a) Venn diagram shows the total number of differentially expressed genes and those that exclusively expressed in osteoblast generated from different origins. b) gene ontology analysis of the common differentially expressed genes of MSCs-differentiated osteoblast which are generated from different origins. c and d) gene set enrichment analysis of the up regulated genes in the osteoblast generated from iPSC-MSCs and ESC-MSCs respectively. e) gene regulatory network for genes exclusively involved in the differentiation of ESC-MSCs into osteoblasts and mapped expression of ESC-MSCs generated osteoblast, f) iPCS-MSCs and g) BM-MSCs derived osteoblast on the regulatory network. h) Ontology analysis of the regulatory network. The cell cycle related terms are colored in red. Majority of the genes in these terms are down regulated.

\section{Supplementary files:}




\section{Supplementary tables:}

Supplementary Table 1. Ontology analysis of the most up regulated genes of NPC grown on 2D substrate. Supplementary Table 2. Ontology analysis of the most up regulated genes of NPC grown on 3D substrate.

Supplementary Table 3. Gene set enrichment analysis of the common differentially expressed genes between $2 \mathrm{D}$ and $3 \mathrm{D}$ scaffold.

Supplementary Table 4. Common differential expressed genes between osteoblasts generated from three different cell of origin.

\section{Supplementary Fig.s:}

Supplementary Fig. 1. PCA Fig. of the osteoblasts generated from three different cell of origin.

Supplementary Fig. 2. Heatmap analysis of all differentially expressed genes in all three comparison.

a

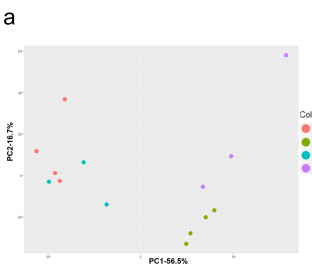

f
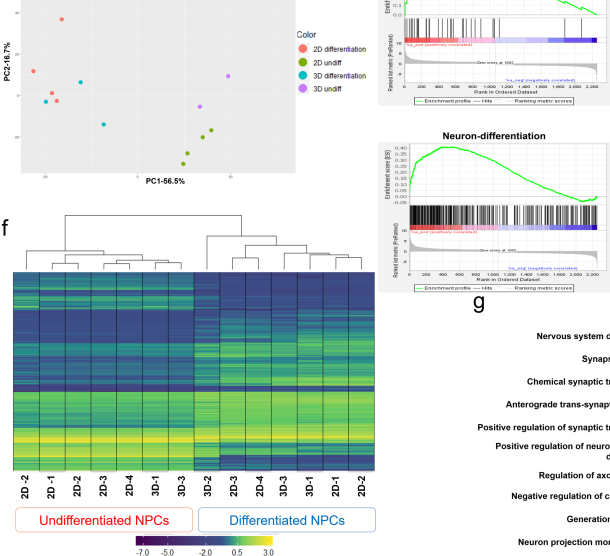

|||||||||||||||||||||||||||||||||||||||||||||||||||-

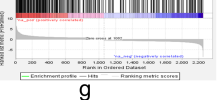

g
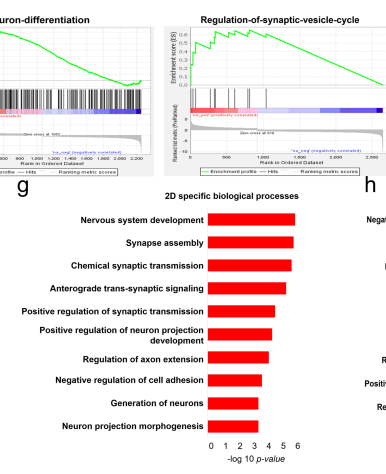

e
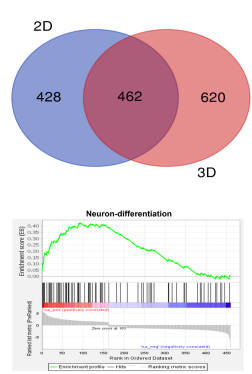

h

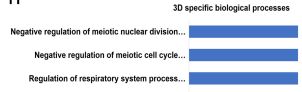

Fatsoluber vitamin catabolic processs
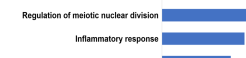

W
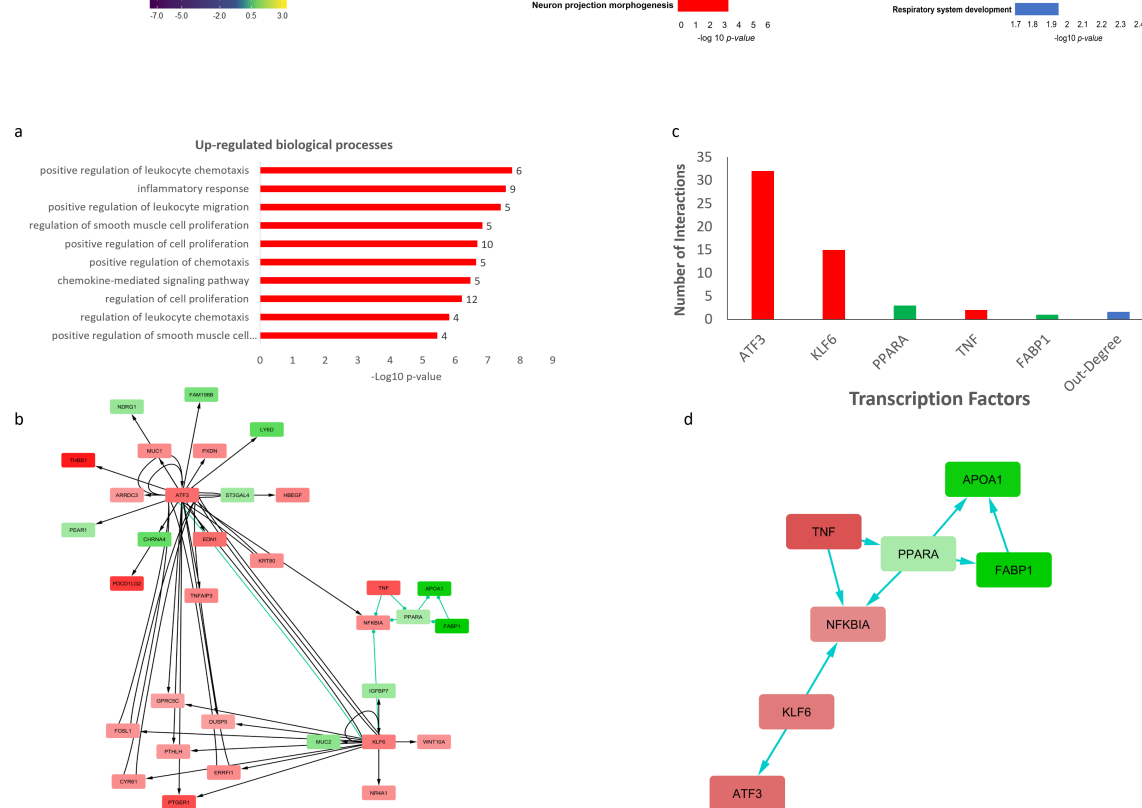
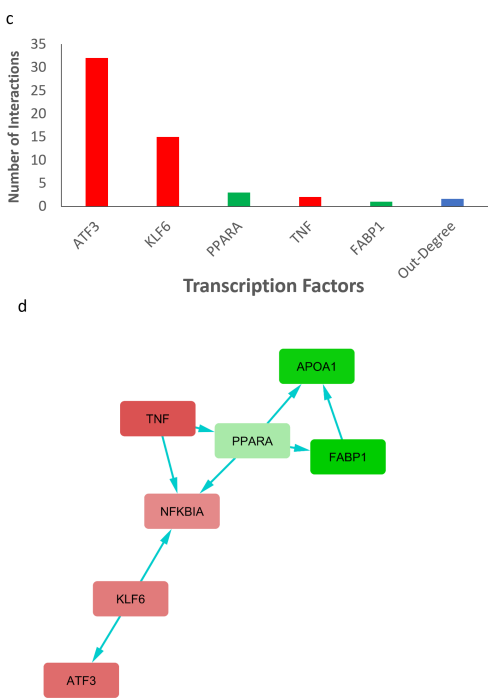

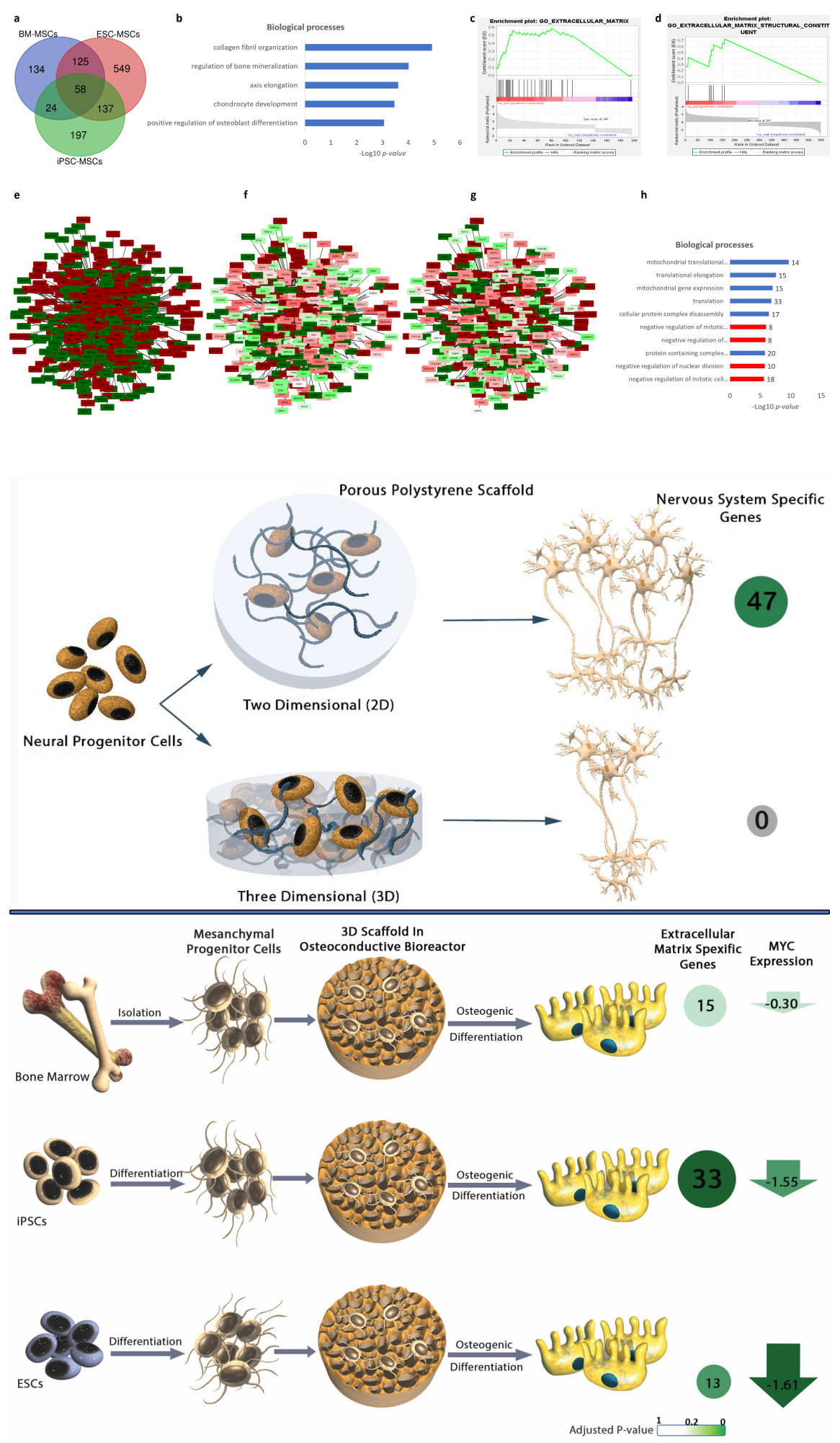